\title{
Hydrodynamical simulations of convection-related stellar micro-variability
}

\section{The enigmatic granulation background of the CoRoT target HD $49933^{\star}$}

\author{
H.-G. Ludwig ${ }^{1, \star \star}$, R. Samadi ${ }^{2}$, M. Steffen ${ }^{3}$, T. Appourchaux ${ }^{4}$, F. Baudin ${ }^{4}$, K. Belkacem ${ }^{5}$, P. Boumier ${ }^{2}$, \\ M.-J. Goupil ${ }^{2}$, and E. Michel ${ }^{2}$ \\ 1 GEPI, Observatoire de Paris, CNRS, Univ. Paris 7, 92195 Meudon Cedex, France \\ e-mail: Hans . Ludwig@obspm. fr \\ 2 LESIA, Observatoire de Paris, CNRS (UMR 8109), Univ. Paris 6, Univ. Paris 7, 92195 Meudon Cedex, France \\ Astrophysikalisches Institut Potsdam, An der Sternwarte 16, 14482 Potsdam, Germany \\ ${ }^{4}$ Institut d'Astrophysique Spatiale, Univ. Paris 11, CNRS (UMR 8617), 91405 Orsay, France \\ 5 Institut d'Astrophysique et de Géophysique de l'Université de Liège, Allée du 6 Août 17, 4000 Liège, Belgium
}

Received 23 February 2009 / Accepted 17 April 2009

\begin{abstract}
Context. Local-box hydrodynamical model atmospheres provide statistical information about a star's emergent radiation field which allows one to predict the level of its granulation-related micro-variability. Space-based photometry is now sufficiently accurate to test model predictions.

Aims. We aim to model the photometric granulation background of HD 49933 as well as the Sun, and compare the predictions to the measurements obtained by the CoRoT and SOHO satellite missions.

Methods. We construct hydrodynamical model atmospheres representing HD 49933 and the Sun, and use a previously developed scaling technique to obtain the observable disk-integrated brightness fluctuations. We further performed exploratory magnetohydrodynamical simulations to gauge the impact of small scale magnetic fields on the synthetic light-curves.

Results. We find that the granulation-related brightness fluctuations depend on metallicity. We obtain a satisfactory correspondence between prediction and observation for the Sun, validating our approach. For HD 49933, we arrive at a significant over-estimation by a factor of two to three in total power. Locally generated magnetic fields are unlikely to be responsible, otherwise existing fields would need to be rather strong to sufficiently suppress the granulation signal. Presently suggested updates on the fundamental stellar parameters do not improve the correspondence; however, an ad-hoc increase of the HD 49933 surface gravity by about 0.2 dex would eliminate most of the discrepancy.

Conclusions. We diagnose a puzzling discrepancy between the predicted and observed granulation background in HD 49933, with only rather ad-hoc ideas for remedies at hand.
\end{abstract}

Key words. convection - hydrodynamics - methods: numerical - stars: atmospheres - stars: late-type - stars: individual: HD 49933

\section{Introduction}

The bright $\left(m_{\mathrm{V}}=5.77\right)$ F-type dwarf HD $49933(\mathrm{HR} 2530$, ADS 5505A) was the first prime seismic target of the CoRoT space photometry mission (Auvergne et al. 2009). A clear signature of solar-like oscillations was detected in the acquired lightcurve (Appourchaux et al. 2008; Michel et al. 2008). This confirms expectations about the presence of solar-like oscillations in HD 49933 which were anticipated from results of ground-based observations of radial velocity (Mosser et al. 2005).

It is now generally accepted that solar-like oscillations are excited by stochastic convective motions taking place in the stellar surface layers on time-scales of the same order as the periods of the excited modes. The convective flows are thermally driven, and imprint a time-varying brightness pattern - granulation - on

\footnotetext{
* The CoRoT space mission, launched on December 27th 2006, has been developed and is operated by CNES, with the contribution of Austria, Belgium, Brazil, ESA, Germany and Spain.

$\star \star$ CIFIST Marie Curie Excellence Team.
}

the stellar surface. While the oscillations manifest themselves as discrete peaks in a temporal power spectrum of a star's lightcurve, the stochastic evolution of the granulation pattern leads to a continuous signal, the so-called granulation background, since it usually constitutes the background on which oscillatory peaks are superimposed in a spectrum. Oscillations, as well as granulation, induce very small brightness fluctuations, challenging the sensitivity level of photometry achievable from the ground, and motivating photometry from space such as that performed by CoRoT.

The modelling of stellar granulation - mainly driven by the need for improved stellar atmosphere models of late-type stars made substantial progress over the last two decades. It has now reached a level that should allow a direct comparison between the predicted and observed granulation properties, in particular the granulation background (Trampedach et al. 1998; Svensson \& Ludwig 2005; Ludwig 2006; Guenther et al. 2008). Hitherto, the comparison was somewhat hampered by the limited photometric accuracy, duty cycle, and duration of existing stellar 
Table 1. CO5 BOLD radiation-hydrodynamics model atmospheres.

\begin{tabular}{lllrllllllllll}
\hline \hline Model & $\begin{array}{l}T_{\text {eff }} \\
{[\mathrm{K}]}\end{array}$ & $\begin{array}{l}\log g \\
{\left[\mathrm{~cm} / \mathrm{s}^{2}\right]}\end{array}$ & {$[\mathrm{Fe} / \mathrm{H}]$} & $\begin{array}{l}R \\
{\left[R_{\odot}\right]}\end{array}$ & $\begin{array}{l}l_{x}, l_{y} \\
{[\mathrm{Mm}]}\end{array}$ & $\begin{array}{l}l_{z} \\
{[\mathrm{Mm}]}\end{array}$ & $\begin{array}{l}T \\
{[\mathrm{ks}]}\end{array}$ & $\begin{array}{l}\tau_{\mathrm{c}} \\
{[\mathrm{s}]}\end{array}$ & $\begin{array}{l}H_{\mathrm{p}}^{\text {surf }} \\
{[\mathrm{Mm}]}\end{array}$ & $\delta I_{\mathrm{rms}} / I$ & $\begin{array}{l}\sigma_{\text {gran }} \\
{[\mathrm{ppm}]}\end{array}$ & $N_{\text {obm }}$ & Modelcode \\
\hline S0 & 6720 & 4.25 & 0.0 & 1.35 & 16.4 & 24.2 & 135.6 & 29.5 & 0.241 & 0.196 & $84(17)$ & 5 & $\mathrm{~d} 3 \mathrm{t} 68 \mathrm{~g} 43 \mathrm{~mm} 00 \mathrm{n} 01$ \\
S1 & 6730 & 4.25 & -1.0 & 1.35 & 16.1 & 24.2 & 73.8 & 29.1 & 0.238 & 0.229 & $64(13)$ & 6 & $\mathrm{~d} 3 \mathrm{t} 68 \mathrm{~g} 43 \mathrm{~mm} 10 \mathrm{n} 01$ \\
Sun1 & 5780 & 4.44 & 0.0 & 1.00 & 5.6 & 2.5 & 52.2 & 17.8 & 0.141 & 0.172 & $40(8)$ & 5 & $\mathrm{~d} 3 \mathrm{gt} 57 \mathrm{~g} 44 \mathrm{n} 53$ \\
\hline
\end{tabular}

"Model" is the model's name used in this paper, $T_{\text {eff }}$ the effective temperature, $\log g$ the gravitational acceleration, $[\mathrm{Fe} / \mathrm{H}]$ the metallicity, $R$ an assumed stellar radius (not intrinsic to the simulation proper), $l_{x}=l_{y}$ the linear horizontal size of the square-shaped computational box, $l_{z}$ its vertical extent, $T$ the duration of the simulated time series, $\tau_{\mathrm{c}}$ the sound crossing time over $H_{\mathrm{P}}^{\text {surf }}, H_{\mathrm{P}}^{\text {surf }}$ the pressure scale height at Rosseland optical depth unity, $\delta I_{\mathrm{rms}} / I$ the relative spatial white light intensity contrast at disk-center, $\sigma_{\text {gran }}$ the temporal relative disk-integrated granulation-related brightness fluctuations in white light (in parenthesis an estimate of the uncertainty), $N_{\mathrm{obm}}$ the number of equivalent frequency points considered in the solution of the radiative transfer equation, "Modelcode" an internal identifier of the model sequence.

measurements. The outstanding accuracy achieved on HD 49933 by CoRoT offers a new opportunity for a stringent comparison between predicted and observed granulation background, and is attempted here. We will see, however, that some problems are encountered.

The paper is organized as follows: we briefly describe the photometric data (Sect. 2), and the hydrodynamical granulation models (Sect. 3), comment on how we derive the synthetic background spectra (Sect. 3.1), present comparisons for the Sun and HD 49933 (Sect. 4), and finish by discussing effects which may play a role in the encountered problems (Sects. 5 and 6).

\section{CoRoT photometric data and power spectra}

We used reduced photometric data of the N2-level (Samadi et al. 2006) covering $137 \mathrm{~d}$ for HD 49933 from long run LRa01, and $157 \mathrm{~d}$ for the F-dwarfs HD 181420 and HD 181906 from long run LRc01. We applied the same bolometric correction (Michel et al. 2009) to all targets, amounting to a factor of 1.11 in amplitude of the brightness fluctuations. We calculated power spectra by applying a standard Fast Fourier Transform from the bolometric light-curve without further processing. In particular, we did not apply any correction to the long-term trend apparent in the light-curve of HD 49933. The reason was that applying the measured loss of instrumental sensitivity due to aging again produced a trend but in the opposite sense. This in turn resulted in a power spectrum almost indistinguishable from the power spectrum of the uncorrected data. On the other hand, simply detrending by removing any linear long-term variation had an impact on the power - but at frequencies $v<0.3 \mathrm{mHz}$ only. These frequencies are not relevant to the conclusions drawn in this paper. Hence, we did not further investigate this issue, but remark that there is an instrument-related uncertainty in the measured power and its slope in the range $v<0.3 \mathrm{mHz}$.

When we refer to "power" in this paper we always mean power spectral density. We follow the normalization that the power integrated over the interval between zero (excluded) and the Nyquist frequency equals the variance $\sigma^{2}$ of the signal in the time domain.

\section{3. $\mathrm{CO}^{5} \mathrm{BOLD}$ radiation-hydrodynamics simulations}

We used the radiation-hydrodynamics code $\mathrm{CO}^{5} \mathrm{BOLD}$ (for further information about the code and applications see Freytag et al. 2002; Wedemeyer et al. 2004) to construct three 3D Cartesian "local-box" model atmospheres. Two models were intended to represent HD 49933, and one - for reference - the Sun. Table 1 summarizes the model properties. The atmospheric parameters for HD 49933 were chosen close to values recommended in the literature, $T_{\text {eff }}=6780 \pm 130 \mathrm{~K}$ (Bruntt et al. 2008), and $\log g \approx 4.25$ (Appourchaux et al. 2008). The metallicity of the star is $[\mathrm{Fe} / \mathrm{H}] \approx-0.37 \mathrm{dex}$ (Solano et al. 2005; Gillon \& Magain 2006). The opacities used in the models for HD 49933 are based on detailed low-temperature opacities from the MARCS stellar atmosphere package (Gustafsson et al. 2008). We only had these data available for particular metallicities, and not for arbitrary element mixtures. Due to this restriction, we decided to compute two 3D models with metallicities which bracket the star's observed metallicity. As will be demonstrated below, the chemical composition has an important impact on the model properties. The solar model Sun 1 is a $\mathrm{CO}^{5} \mathrm{BOLD}$ model of an older generation employing opacities from the ATLAS model atmosphere package (Kurucz 2005). While this leads to some systematic differences between the models, we believe that they so not influence the comparisons performed in this paper. Our two hydrodynamical model atmospheres for HD 49933 were also employed to predict excitation rates of oscillatory modes (Samadi et al. 2008, 2009).

Unfortunately, the aforementioned stellar parameters are not completely reliable. Bruntt et al. (2004) list effective temperatures and surface gravities from various determinations which differ by almost $500 \mathrm{~K}$ and $0.5 \mathrm{dex}$, and the debate about the exact parameters is still ongoing (Bruntt 2009; Kallinger et al. 2008). It may turn out that HD 49933 is $200-300 \mathrm{~K}$ cooler than assumed in our models. More important for the level of the granulation background, however, is the the value of the surface gravity. We will come back to this point later but want to emphasize that HD 49933 has an accurately known parallax of $33.7 \pm 0.4$ mas from HIPPARCOS (van Leeuwen 2007 which helps to constrain its surface gravity and stellar radius. In this paper, we use a radius of $1.35 R_{\odot}$ for HD 49933 , within the error box given by Appourchaux et al. (2008) of $1.36 \pm 0.06 R_{\odot}$.

Since the brightness fluctuations which are central to the present investigation are intimately linked to the heating and cooling provided by the radiation field to the gas, we give here some details about the treatment of the radiative transfer in the hydrodynamical models. All models employ $140 \times 140 \times$ 150 grid points for the spatial mesh in the $x$-, $y$-, and (vertical) $z$-direction, respectively. For all models, the mesh spacing is equidistant in the horizontal directions. In vertical direction, S0 and S1 employ a non-equidistant spacing, Sun 1 again an equidistant mesh. In the important layers around $\tau=1$ the spacing in vertical direction amounts to $46.9 \mathrm{~km}\left(0.19 H_{\mathrm{P}}^{\text {surf }}\right.$, definition see Table 1$), 48.2 \mathrm{~km}\left(0.20 H_{\mathrm{P}}^{\text {surf }}\right)$, and $15.1 \mathrm{~km}\left(0.11 H_{\mathrm{P}}^{\text {surf }}\right)$ for models S0, S1, and Sun1, respectively. Note that the models S0 and $\mathrm{S} 1$ use the same vertical mesh. The difference in resolution at 
$\tau=1$ is the result of how the model adjusts on the computational mesh. The radiative transfer is solved along long characteristics employing Feautrier's method (e.g., Mihalas 1978) assuming strict LTE. Corresponding "rays" are started at each point of the spatial mesh in the top-most layer. All models use bundles of rays in one vertical and two inclined directions, as well as four azimuthal directions $(M=3$ case described in Ludwig 2006) coinciding with the coordinate axes. In total this amounts to 176400 rays along which the radiative transfer equation is solved at each time step. The number of equivalent wavelength points $\mathrm{N}_{\mathrm{obm}}$ to represent the wavelength dependence of the radiation field is five for models S0, and Sun1, six for S1, as given in Table 1. The necessary interpolation of the radiative heating or cooling between the system of rays and spatial mesh (for hydrodynamics) is performed in an energy-conserving fashion. To optimize performance, the treatment of the radiative transfer is switched to the diffusion approximation in the deep, optically thick layers.

Finally, we want to point out two aspects which are of particular importance for the interpretation of the granular background. Qualitatively, the lower metallicity in model S1 in comparison to S0 leads to a lower overall opacity (primarily due to the lower $\mathrm{H}^{-}$opacity at lower electron pressure). This in turn implies an increase of the mass density at given optical depth. At optical depth unity, we find an increase of the mass density by $\approx 40 \%$ in model $\mathrm{S} 1$ relative to S0. At fixed flow geometry, the higher density would require a smaller temperature contrast or smaller velocity differences between up- and down-flowing material to transport the prescribed stellar energy flux. What actually happens in S1 is an increase of the temperature contrast reflected by an increase of the intensity contrast (see Table 1). This is likely a consequence of the dependence of the opacity on temperature. On the other hand, as expected, the typical velocity amplitudes in model $\mathrm{S} 1$ are smaller by about $\approx 10 \%$ at the maximum of the vertical velocity. Finally, the typical granular scales become shorter in S1. The combination of these factors determines the amplitude and characteristic frequency of the granulation background.

\subsection{Synthetic power spectra of the observable flux}

The hydrodynamical models provide time series of the radiation intensity at different limb-angles including the temporal convection-related fluctuations. Ludwig (2006) showed that, together with an assumption about the stellar radius, this information is sufficient to predict the power spectrum of the diskintegrated, observable, relative brightness variations. He obtained for the frequency component of the power spectrum the relation

$$
\frac{\left\langle\hat{f} \hat{f}^{*}\right\rangle}{\langle f\rangle^{2}}=N^{-1} \frac{\sum_{m=1}^{M} c_{m} \mu_{m}^{2}\left\langle\hat{I}_{m} \hat{I}_{m}^{*}\right\rangle}{\left(\sum_{m=1}^{M} c_{m} \mu_{m}\left\langle I_{m}\right\rangle\right)^{2}}
$$

where

$N=\frac{2 \pi R^{2}}{A}$.

Angular brackets denote expectation values, $f$ the observable disk-integrated flux, and $\hat{f}$ its (complex) Fourier transform. The asterisk indicates the conjugate complex, $c_{m}$ a discrete integration weight, $\mu_{m}$ the cosine of the limb-angle, and $I_{m}$ the spatial average of the intensity in direction cosine $\mu_{m}$. According Eq. (1) the observable power scales inversely proportional to the number $N$ of simulation boxes of surface area $A$ tiling the visible stellar hemisphere of radius $R$. For a detailed derivation and discussion of Eq. (1) see Ludwig (2006).

Due to the finite duration of a simulation, the obtained power spectra of the relative brightness fluctuations exhibit appreciable noise. To improve the effective signal-to-noise level and facilitate the comparison to observations we fit a simple analytical model to the simulated spectra. The analytical model reflects our prejudice that the frequency dependence of the granulationrelated background signal should show rather little structure. Moreover, it allows us to eliminate the acoustic eigenmodes of the computational domain which are excited by the convectionrelated fluctuations but are not directly comparable to the observed modes. We applied a model for the spectral power density $P$ which is a generalized Harvey model (Harvey 1985) for the background, plus a sum of Lorentzians for the box-modes according to

$P(v)=\frac{b}{1+\left(\frac{v}{v_{1}}\right)^{\alpha_{1}}+\left(\frac{v}{v_{2}}\right)^{\alpha_{2}}}+\sum_{k=1}^{K} \frac{a_{k}}{1+\left(\frac{v-u_{k}}{w_{k}}\right)^{2}}$.

$v$ is the cyclic (temporal) frequency. All other variables are fitting parameters of the model: $b$ is the asymptotic amplitude of the background towards low frequencies, $v_{1}$ and $v_{2}$ characteristic frequencies of the background, $\alpha_{1}$ and $\alpha_{2}$ power law exponents, $a_{k}$ are the mode amplitudes of $K$ considered modes, $u_{k}$ their frequency positions, $w_{k}$ their line widths. We performed a maximum likelihood estimation of the parameters; we used the commonly adopted model that each frequency component of a power spectrum is statistically independent and follows a probability distribution $p$ which is a $\chi^{2}$-distribution with two degrees of freedom. This is an exponential of the form

$p(x)=\frac{1}{\langle x\rangle} \exp \left(-\frac{x}{\langle x\rangle}\right)$

$\langle x\rangle$ is the expectation value of the random variable $x$. The model (3) allows us to extrapolate the fitted spectrum to frequencies higher than the Nyquist frequency $v_{\text {Nyquist }}$. To mitigate effects of aliasing present at the highest frequencies of the simulated spectra, we did not fit $P(v)$ itself but $P(v)+P\left(2 v_{\text {Nyquist }}-v\right)$. This includes the "mirroring" of high frequency power into the frequency domain of interest by aliasing. As we will see, $P(v)$ decreases strongly towards high frequencies so that aliasing effects are only noticeable close to the Nyquist frequency.

To maximize the likelihood function we used the POWELL function implemented in IDL. The maximization problem turned out not to be well conditioned, and some manual intervention was always necessary to obtain a stable and acceptable fit. This points to the need for a more sophisticated fitting procedure with suitable regularization. However, for the present purposes we considered the fitting rather a kind of constrained smoothing so that the shortcomings from the viewpoint of statistical stringency were not considered vital. We verified that our fitting resulted in a representation close to what was obtained by direct smoothing of the simulated spectra.

\section{Results}

\subsection{Fitting the simulated raw spectra}

Figure 1 illustrates the result of fitting the analytical model of Eq. (3) to the simulated raw spectra calculated from the time series with the help of Eq. (1). Table 2 lists the fitted parameters. As already mentioned, the simulated spectra show an appreciable level of noise. In the figure, the simulated spectra were 


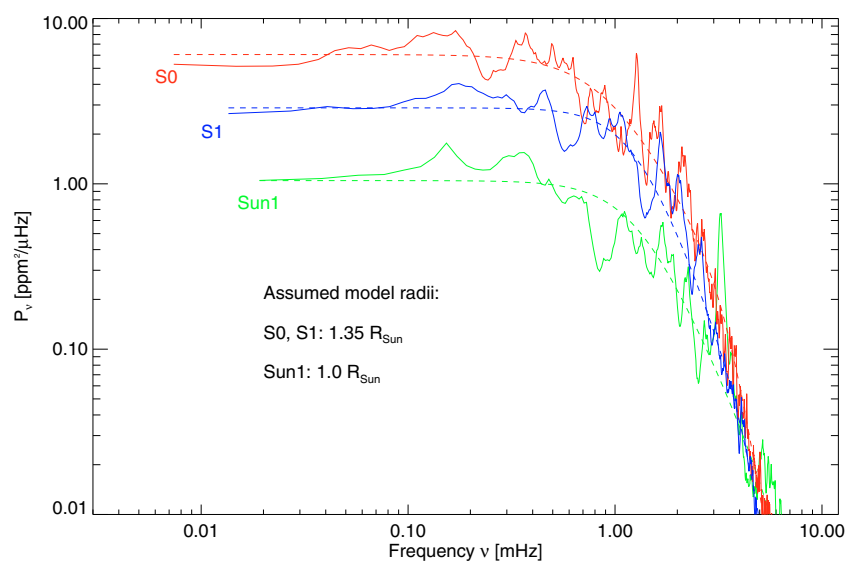

Fig. 1. Inter-comparison of the raw (solid lines) and fitted (dashed lines) spectra (power spectral density) of the three hydrodynamical simulations. The contribution of box-modes to the fitted signal was left out in the graphs.

Table 2. Fitted parameters of Eq. (3) for the synthetic power spectra of the three hydrodynamical models.

\begin{tabular}{llll}
\hline \hline \multirow{2}{*}{ Parameter } & \multicolumn{3}{c}{ Hydrodynamical models } \\
& S0 & S1 & Sun1 \\
\hline$b$ & 6.056 & 2.881 & 1.046 \\
$v_{1}$ & 0.9593 & 1.259 & 1.295 \\
$\alpha_{1}$ & 2.486 & 3.434 & 2.953 \\
$v_{2}$ & 2.383 & 2.821 & 3.667 \\
$\alpha_{2}$ & 8.061 & 9.278 & 8.035 \\
$a_{1}$ & 0.4130 & 0.5389 & 2.149 \\
$u_{1}$ & 1.266 & 1.674 & 3.252 \\
$w_{1}$ & 0.09507 & 0.08464 & 0.02861 \\
$a_{2}$ & 4.498 & 1.732 & \\
$u_{2}$ & 1.676 & 2.027 & \\
$w_{2}$ & 0.01155 & 0.02994 & \\
$a_{3}$ & 2.188 & 2.142 & \\
$u_{3}$ & 2.107 & 2.618 & \\
$w_{3}$ & 0.01217 & 0.004683 & \\
\hline
\end{tabular}

Background- and line-amplitudes are given in $\mathrm{ppm}^{2} / \mu \mathrm{Hz}$, frequencies in $\mathrm{mHz}$. Missing values for a line indicate that it was left out since unnecessary to obtain a satisfactory fit.

smoothed to allow a clearer comparison. By comparing solar models with different numerical set-ups, we estimated that the absolute uncertainty in the simulated spectra amounts to about $\pm 20 \%$ in total (i.e. frequency integrated) amplitude; we expect the relative accuracy among models of similar numerical setups to be somewhat better. Note that we left out the contribution of the box modes in the fitting functions in Fig. 1. The simulated spectra show clear differences in the total power between the Sun and HD 49933 - primarily reflecting the change of the granular cell size relative to the stellar radius among the stars. The metal-depleted model for HD 49933 S1 shows only 58\% of the model SO in total power. This is the result of a reduction of the granular scale at lower metallicity, over-compensating of the increase in the contrast (cf. Eq. (5)). Moreover, the power in model $\mathrm{S} 1$ is shifted towards lower frequencies. This we trace back to longer granular life-times in S1, presumably related to the lower typical velocities in S1. All models predict a rather steep drop of the granular background signal towards high frequencies, similar to the one observed in the Sun.

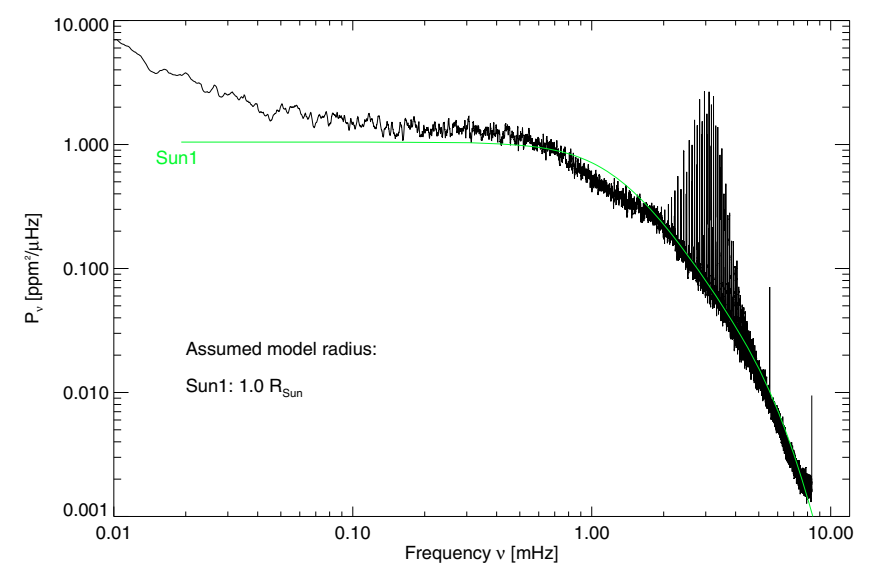

Fig. 2. Power spectra of disk-integrated photometric fluctuations for the Sun: the predicted background signal of model Sun1 (green/grey solid line) and observational data from SOHO/VIRGO (black solid line). Note the steep decline in power towards high frequencies.

\subsection{Comparison with VIRGO observations of the sun}

Figure 2 constitutes an update of results presented by Svensson \& Ludwig (2005), and shows a comparison (not fit) between the disk-integrated, photometric fluctuations derived from the solar model Sun1, and observational data from the VIRGO instrument on board the SOHO satellite. Our focus is the high frequency region of the solar signal in which granular contributions dominate. The VIRGO power spectrum was calculated from (level 2) time series data provided by the VIRGO team. One year of data (1996.5-1997.5) was chosen close to solar minimum activity to minimize the possible contribution of activity related variability. The plotted power spectrum is based on data of the green channel of the VIRGO three-channel sunphotometer (SPM, see Fröhlich et al. 1997). Following the procedure of Svensson \& Ludwig (2005), it has been converted to white light fluctuations by matching (by shifting in power) a corresponding power spectrum based on VIRGO PMO6V-A absolute radiometer data in the frequency range 0.3 to $2.0 \mathrm{mHz}$. Instead of using the PMO6V-A power spectrum directly, this rather involved procedure was necessary since PMO6V-A and SPM power spectra deviate substantially in the high frequency region. The authors found little information about this mismatch in the literature. However, it appears to be agreed that the SPM spectrum reflects the actual solar behavior (Fröhlich et al. 1997; Andersen et al. 1998), in particular showing the steep (roughly as $v^{-4}$ ) decline at the highest frequencies.

We find a satisfactory agreement of the continuous background signal between model predictions and observations between 0.1 and $8.0 \mathrm{mHz}$. In particular, the background in the p-mode frequency range is matched quite well. The observed background power shows a "kink" at frequencies slightly above $1 \mathrm{mHz}$ relative to the model prediction, and the "plateau" power around $0.2 \mathrm{mHz}$ is not exactly represented. Michel et al. (2009) interpreted the "kink" as the sign of the transition between a meso-granular and granular contribution to the power spectrum. This interpretation is obviously not supported by our granulation model, which already provides too much power around $1 \mathrm{mHz}$, and has a geometrical dimension which would not allow the development of meso-granular convection cells of 5-10 Mm size (November et al. 1981). Moreover, Michel and co-workers attribute a similar total fluctuation amplitude to meso-granulation and granulation. According to Eq. (5), this would correspond to a spatial brightness contrast which would be a sizable fraction 
of the granulation contrast and should be easily recognizable in images of the solar surface - which is not the case.

Towards low frequencies $(v<0.1 \mathrm{mHz})$, the increase of the observed signal is due to the solar magnetic activity, and cannot be represented by the pure (non-MHD) hydrodynamical model applied here. We also considered the power spectrum at solar maximum (year 2000.5-2001.5, not shown). Towards solar maximum, the activity-related power increases but leaves the granulation-related signal intact; the power spectrum is essentially identical at frequencies $v>0.2 \mathrm{mHz}$. It is difficult to provide an objective criterion to determine to which lowest temporal frequency our model can make reliable predictions. A limit is set by the maximum size that features can develop in the simulation. However, it is not obvious how this relates to their life-time, hence the frequencies on which they will have an impact.

A conspicuous feature exhibited by the observations as well as the model is the "step-like" shape of the granular background signal. As it will be shown later, this feature is not obvious in the observations of HD 49933, while it is predicted by the simulations.

\subsection{Comparison with CoRoT observations of HD 49933}

Figure 3 shows a comparison between the predicted power spectra (power spectral density) of models S0 and S1. Ideally, the observed spectrum should fall somewhere between models S0 and S1. This is not at all the case; the predictions lie noticeably higher with a power almost a factor of two greater than observed around $0.9 \mathrm{mHz}$. Moreover, the overall shape of the observed spectrum is not well represented by the models. In particular, the conspicuous drop of the background level is not obvious in the observations.

In Fig. 3 we added two spectra of F-dwarfs which were also observed by CoRoT. The stars have parameters not too different from HD 49933 (HD 181420: $T_{\text {eff }}=6650 \mathrm{~K}, \log g=4.17$, $[\mathrm{M} / \mathrm{H}]=-0.04$; HD 181906: 6380/4.15/-0.14; see Michel et al. 2008). The overall levels of the brightness fluctuations differ, however, the spectra show a remarkable similarity in shape. We take this as an indication that HD 49933 is a typical representative of its class, and not an exceptional case among F-dwarfs. We note here that the similarity of the spectral shape at $v>0.3 \mathrm{mHz}$ might be connected to the rather similar rotational periods of the stars of 3.4d for HD 49933 (Appourchaux et al. 2008), $2.8 \mathrm{~d}$ for HD 181906 (García et al. 2009), and 3.5 . . 4.5 d for HD 181420 (Barban et al. 2009).

Michel et al. (2008) assumed that the continuum in the p-mode region indeed corresponds to the granulation background, fitted it with a standard Harvey model, and arrived at granulation-related brightness fluctuations of $\sigma_{\text {gran }} \approx 40 \mathrm{ppm}$ with appreciable uncertainty. This would put the fluctuations in HD 49933 on the same level as observed in the Sun, and is significantly lower than our predictions. However, accepting for the moment a much lower level of fluctuations, we experimented in an ad-hoc fashion by adding (besides photometric noise) an activity-like signal (assuming a power-law) to our simulated granulation spectra, and simultaneously scaled their power and characteristic frequency to enforce correspondence with the observations. Figure 4 illustrates the outcome after scaling the power derived from the models by a factor of 0.33 and the frequency by a factor of 1.6. This resulted in a plausible albeit not entirely satisfactory - correspondence. Later we shall try to interpret the scaling in terms of a change of fundamental stellar parameters (see Sect. 5.1).

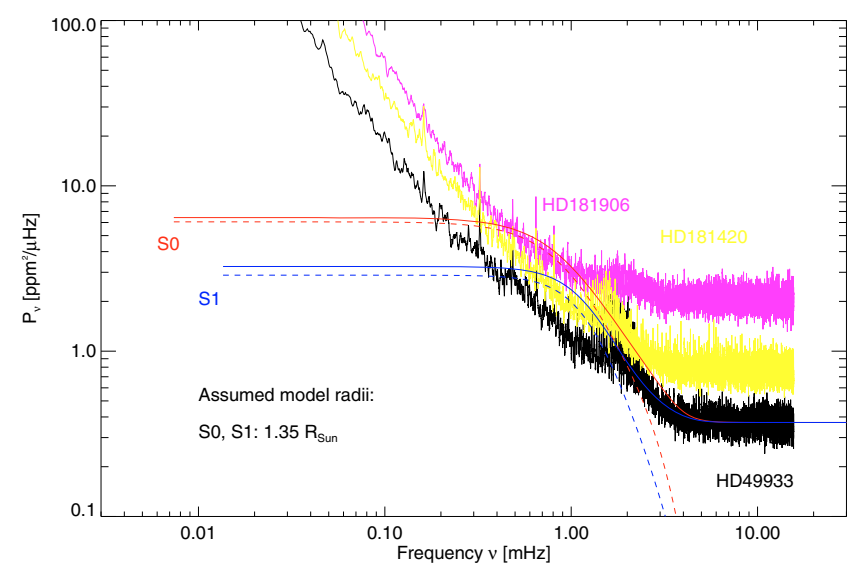

Fig. 3. Power spectra for HD 49933: dashed lines depict the predictions from models S0 and S1. The solid lines are again the predicted spectra but increased by a constant corresponding to the observed photometric white noise level. The black line depicts the spectrum of HD 49933. The other two solid lines depict spectra of the similar F-dwarfs HD 181420 and HD 181906.

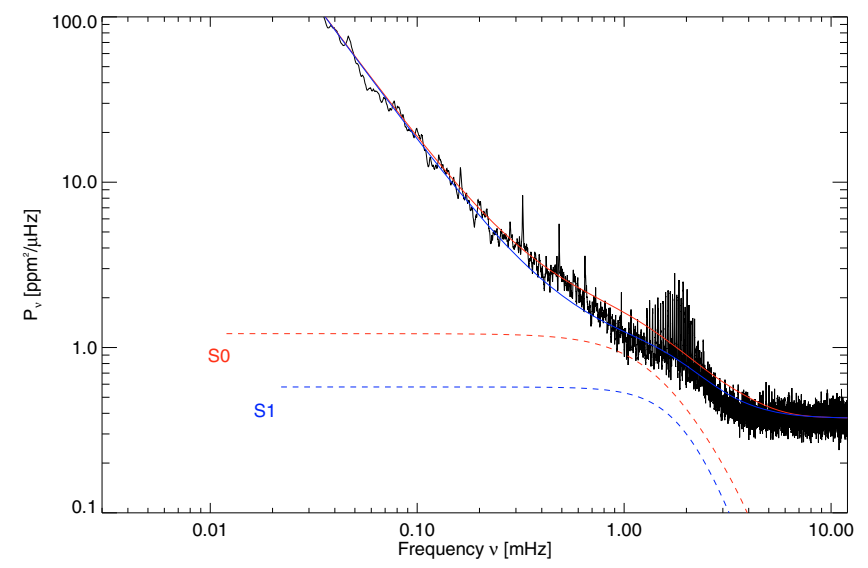

Fig. 4. Like Fig. 3, but with scaled models S0 and S1 (for details see text) and adding an ad-hoc signal for magnetic activity and photometric noise (solid lines). The dashed lines depict the models after scaling without added ad-hoc components.

\section{Discussion}

Can we find a reason for the mismatch between model and observation for HD 49933? In the following we discuss the impact of uncertainties in the fundamental parameters and the influence of magnetic activity on the mismatch.

\subsection{Uncertainties in the fundamental parameters}

As alluded to before, the fundamental parameters of HD 49933 (in particular $T_{\text {eff }}, \log g$, and $R$ ) might be noticeably different from the values we assumed in our modeling. Ludwig (2006) give an approximate relation between the spatial contrast of the granulation and the photocentric variability, which can be equivalently stated in terms of the relative temporal (rms) brightness fluctuations $\sigma_{\text {gran }}$ as

$\sigma_{\text {gran }} \propto \frac{l_{\text {gran }}}{R} \frac{\delta I_{\mathrm{rms}}}{\bar{I}}$

$l_{\text {gran }}$ is the typical linear size of a granular cell, $R$ the stellar radius, $I$ the (spatially resolved) emergent intensity, $\delta I_{\text {rms }}$ its spatial 
standard deviation, and $\bar{I}$ its spatial average. We wrote a proportionality only since we want to investigate the differential functional dependence on the fundamental parameters here. Freytag et al. (1997) argue on the basis of numerical simulations that $l_{\text {gran }}$ is proportional to the pressure scale height at the stellar surface $H_{\mathrm{P}}^{\text {surf }}$

$l_{\text {gran }} \propto H_{\mathrm{P}}^{\text {surf }} \propto \frac{T_{\text {eff }}}{g}$.

When writing the last proportionality, we assumed a constant mean molecular weight. For the total (frequency integrated) power $\sigma_{\text {gran }}^{2}$ of the brightness fluctuations, it follows that using the fundamental relationship between luminosity $L$, radius, and effective temperature

$\sigma_{\mathrm{gran}}^{2} \propto \frac{T_{\mathrm{eff}}^{2}}{g^{2} R^{2}}\left(\frac{\delta I_{\mathrm{rms}}}{\bar{I}}\right)^{2} \propto \frac{T_{\mathrm{eff}}^{6}}{g^{2} L}\left(\frac{\delta I_{\mathrm{rms}}}{\bar{I}}\right)^{2}$.

Introducing the luminosity in the second proportionality in Eq. (7) was motivated by the notion that the accurately known parallax and visual magnitude of HD 49933 essentially fixes its luminosity. Relation (7) is not exact since the scaling of the granular size with stellar parameters is not exactly accounted for; for instance, the formula does not accurately represent the scaling of $\sigma_{\text {gran }}$ among the models as given in Table 1. However, we think it is good enough to provide an estimate of the differential effects in vicinity of the stellar parameters we used in our modeling. As evident from Table 1, the granulation contrast does not sensitively depend on atmospheric parameters among F- and G-dwarfs, making it largely invariant over the discussed interval of temperatures, gravities, and chemical compositions for HD 49933. Keeping the luminosity fixed gives estimates of the change of $\sigma_{\text {gran }}^{2}$ by +0.59 dex with the recently suggested parameters of Kallinger et al. (2008) $\left(T_{\mathrm{eff}}=6450 \mathrm{~K}, \log g=3.9\right)$, and +0.06 dex with the ones of Bruntt 2009 ("evolutionary solution" $\left.T_{\text {eff }}=6570 \mathrm{~K}, \log g=4.19\right)$. Despite the high exponent of $T_{\text {eff }}$ in Eq. (7), the changes are primarily driven by the changes in $\log g$. We are left with the situation that the suggested parameters would increase the discrepancy between models and observations when applied in the modeling. For the set of parameters suggested by Kallinger and co-workers the deterioration would be rather drastic.

The ad-hoc scaling in power and frequency described in Sect. 4.3 to enforce the correspondence to observations was motivated by Eq. (7), and by the notion that the granular life-time scales with the atmospheric acoustic cut-off frequency (Kjeldsen \& Bedding 1995; Svensson \& Ludwig 2005) as $v \propto g / \sqrt{T_{\text {eff }}}$. This allows us to associate the scaling factors in power and characteristic frequency with changes of $-200 \mathrm{~K}$ in $T_{\text {eff }}$ and +0.2 dex in $\log g$ of HD 49933 (assuming constant luminosity). Again, while helping to reduce the present discrepancies, in particular the increase of the gravity is at odds with the latest determinations of the star's fundamental parameters.

\subsection{Influence of magnetic activity}

By analogy to the rise of power towards low frequencies due to magnetic activity in the Sun (Fig. 2), one is tempted to attribute the rise of power seen in HD 49933 to magnetic activity as well. This is quite plausible, since the light-curve of HD 49933 exhibits clear signatures of spottedness (Mosser, private commun.). Moreover, HD 49933 is a rather rapid rotator, favoring dynamo action. However, polarimetric observations (with NARVAL and

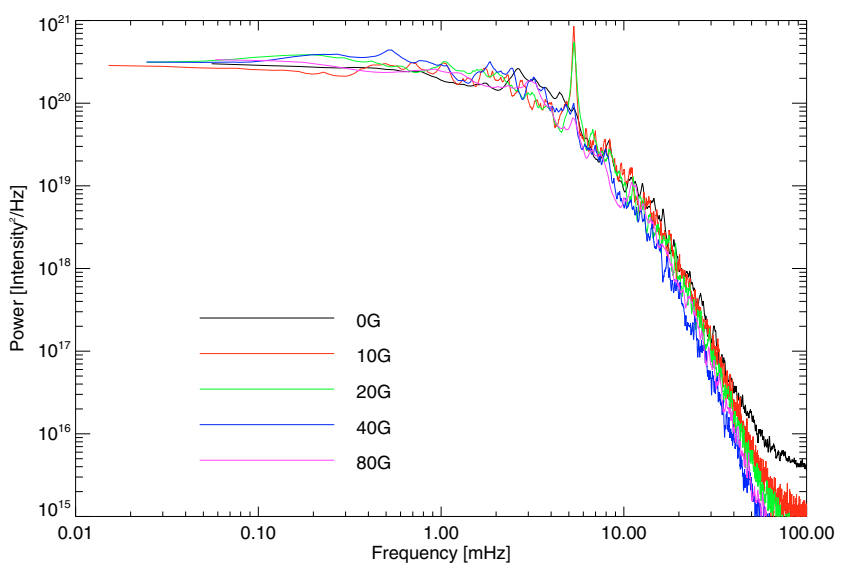

Fig. 5. Temporal power spectra of the horizontally averaged emergent intensity in vertical direction of different 2D MHD runs assuming different levels of initial field strength (solid lines of different colors/shades of grey). The simulations were performed assuming solar atmospheric parameters.

ESPADONS) indicate that there is no magnetic field present above $\approx 1 \mathrm{G}$ which is structured on spatial scales of the order of the stellar radius (Catala et al., in prep.). This raises the question of what kind of mechanism could produce the apparently ubiquitous field on smaller scales?

There is mounting theoretical and observational evidence (Cattaneo 1999; Trujillo Bueno et al. 2004; Vögler \& Schüssler 2007) that dynamo action is possible in convective surface flows generating magnetic fields on very small scales. To investigate whether such magnetic fields can explain the puzzling shape of the spectrum around $1 \mathrm{mHz}$ in HD 49933, we performed exploratory MHD simulations with CO ${ }^{5}$ BOLD (Steffen et al. 2009). They were restricted to two spatial dimensions, and performed for solar atmospheric conditions. We calculated several runs with different, prescribed magnetic flux levels corresponding to field-free to weak plage regions. Figure 5 shows temporal power spectra of the light curves of the simulation runs (not scaled to full disk). Despite that the simulations were tailored to solar conditions, we think that the very similar topology of granular flows among solar-type stars makes them qualitatively also applicable to HD 49933. The power spectra of the lightcurves show a trend towards lower power at higher frequencies for increasing total magnetic flux. Obviously, small scale magnetic fields alter the convective dynamics on small spatial scales but do not lead to the formation of structures on large scales which may leave an imprint at lower frequencies in the brightness fluctuations. The results are preliminary, and further work is necessary. However, for the time being we consider it unlikely that local dynamo action in the granular flow - if at all present can explain the shape of the power spectrum of HD 49933 in the $\mathrm{p}$-mode frequency region.

Equation (5) shows that a reduction of the granular scale at fixed stellar radius could reduce the observed granulation-related brightness fluctuations. It is known that magnetic fields of sufficient strength have this effect (for a recent example see Jacoutot et al. 2008, and references therein). However, to obtain a sizable reduction one would need a mean field strength of several $100 \mathrm{G}$. A local dynamo appears unlikely to sustain such magnetic flux levels (Vögler \& Schüssler 2007), which leaves us with the need for an efficient generation of magnetic fields on larger scales. 


\section{Conclusions}

3D hydrodynamical model atmospheres can be applied to predict granulation-related disk-integrated brightness fluctuations. We demonstrated that the theoretical approach successfully reproduces the convection-related background signal in the frequency region of the observed solar p-modes. Unexpectedly, we found a mismatch between predicted and observed background when applying the same approach to HD 49933, which we argued is a normal representative among the F-dwarfs observed by CoRoT. We excluded local dynamo action in the granular flow as the culprit for the mismatch. The existence of a magnetic field for other reasons with a large filling factor and sufficient strength (several $100 \mathrm{G}$ ) able to noticeably influence the granular dynamics could qualitatively explain the observed low level of granular brightness fluctuations. Whether this is viable from the viewpoint of dynamo theory is unclear. In this context we discussed the observational finding that the star, while magnetically rather active, does not exhibit a magnetic field organized on scales comparable to the stellar radius (Catala et al., in prep.).

Recently suggested new sets of fundamental stellar parameters for HD 49933 (Kallinger et al. 2008; Bruntt 2009) are unlikely to remove the discrepancy - primarily since they suggest a lower surface gravity than we assumed in the present modelling. In contrast, when increasing the gravity by 0.2 dex (to about $\log g=4.45)$, and decreasing the effective temperature by $200 \mathrm{~K}$ (to about $6550 \mathrm{~K}$ ) we can enforce a plausible, albeit not perfect, correspondence between theory and observation. Whether this is compatible with constraints from stellar structure, 3D atmospheric modelling, and p-mode excitation needs to be assessed. For the moment we are left with a puzzling discrepancy between the predicted and observed granulation background in HD 49933, with only a rather ad-hoc idea for a remedy at hand.

The granulation-related brightness fluctuations are sensitive to the stellar metallicity. A reduction of the metallicity from solar to $1 / 10$ solar reduced the total fluctuation power by a factor of 0.58 at the studied $T_{\text {eff }}$ and $\log g$.

If in the future one is able to devise an observational or theoretical calibration of the granular background signal, its high sensitivity to the surface gravity could render it an effective gravimeter. CoRoT has obtained high-precision photometry for many giants. The stronger convection-related background signal makes this easy to detect and quantify. We consider giants as additional, interesting test cases for our understanding of the dynamics of convective flows on the surface of stars.

Acknowledgements. The authors thank Michel Auvergne for providing information on the change of instrumental sensitivity, and Hans Bruntt for communicating his latest results on the fundamental parameters of HD 49933 before publication. H.G.L. would like to thank Claude Catala for discussions about spectroscopy and polarimetry of HD 49933 on several occasions. H.G.L. acknowledges financial support from EU contract MEXT-CT-2004-014265 (CIFIST).

\section{References}

Andersen, B., Appourchaux, T., Crommelynck, D., et al. 1998, in Sounding Solar and Stellar Interiors, ed. J. Provost, \& F.-X. Schmider (Kluwer Academic Publishers), IAU Symp. 181, 147

Appourchaux, T., Michel, E., Auvergne, M., et al. 2008, A\&A, 488, 705

Auvergne, P., Bodin, P., Boisnard, L., et al. 2009, A\&A, 506, 411

Barban, C., Deheuvels, S., Baudin, F., et al. 2009, A\&A, 506, 51

Bruntt, H. 2009, A\&A, 506, 235

Bruntt, H., Bikmaev, I. F., Catala, C., et al. 2004, A\&A, 425, 683

Bruntt, H., De Cat, P., \& Aerts, C. 2008, A\&A, 478, 487

Cattaneo, F. 1999, ApJ, 515, L39

Freytag, B., Holweger, H., Steffen, M., \& Ludwig, H.-G. 1997, in Science with the VLT Interferometer, ed. F. Paresce (Springer), 316

Freytag, B., Steffen, M., \& Dorch, B. 2002, AN, 323, 213

Fröhlich, C., Andersen, B. N., Appourchaux, T., et al. 1997, Sol. Phys., 170, 1

García, R. A., Régulo, C., Samadi, R., et al. 2009, A\&A, 506, 41

Gillon, M., \& Magain, P. 2006, A\&A, 448, 341

Guenther, D. B., Kallinger, T., Gruberbauer, M., et al. 2008, ApJ, 687, 1448

Gustafsson, B., Edvardsson, B., Eriksson, K., et al. 2008, A\&A, 486, 951

Harvey, J. 1985, in Future Missions in Solar, Heliospheric \& Space Plasma Physics, ed. E. Rolfe, \& B. Battrick, ESA SP, 235, 199

Jacoutot, L., Kosovichev, A. G., Wray, A., \& Mansour, N. N. 2008, ApJ, 684, L51

Kallinger, T., Gruberbauer, M., Guenther, D. B., Fossati, L., \& Weiss, W. W. 2008, A\&A, submitted

Kjeldsen, H., \& Bedding, T. 1995, A\&A, 293, 87

Kurucz, R. L. 2005, MSAIt Suppl., 8, 14

Ludwig, H.-G. 2006, A\&A, 445, 661

Michel, E., Baglin, A., Auvergne, M., et al. 2008, Science, 322, 558

Michel, E., Samadi, R., Baudin, F., et al. 2009, A\&A, 495, 979

Mihalas, D. 1978, Stellar Atmospheres (Freeman and Company)

Mosser, B., Bouchy, F., Catala, C., et al. 2005, A\&A, 431, L13

November, L. J., Toomre, J., Gebbie, K. B., \& Simon, G. W. 1981, ApJ, 245, L123

Samadi, R., Fialho, F., Costa, J. E. S., et al. 2006, ESA SP 1306, 317, corrected in [arXiv:astro-ph/0703354]

Samadi, R., Belkacem, K., Goupil, M.-J., Ludwig, H.-G., \& Dupret, M.-A. 2008, Commun. Asteroseismol., 157, 130

Samadi, R., Ludwig, H.-G., Belkacem, K., Goupil, M.-J., \& Dupret, M.-A. 2009, A\&A, submitted

Solano, E., Catala, C., Garrido, R., et al. 2005, AJ, 129, 547

Steffen, M., Ludwig, H. G., \& Steiner, O. 2009, in Proc. IAU Symp. 259, Cosmic Magnetic Fields: from Planets, to Stars and Galaxies, ed. K. G. Strassmeier, A. G. Kosovichev and J. E. Beckman, 233

Svensson, F., \& Ludwig, H.-G. 2005, in Stellar Systems, and the Sun, ed. F. Favata, G. Hussain, \& B. Battrick (ESA Publications Devision), Proceedings of the 13th Cambridge Workshop on Cool Stars, ESA SP 560, 979 ,

Trampedach, R., Christensen-Dalsgaard, J., Nordlund, Å., \& Stein, R. 1998, in The First MONS Workshop: Science with a Small Space Telescope, ed. H. Kjeldsen, \& T. Bedding (Aarhus Universitet), 59

Trujillo Bueno, J., Shchukina, N., \& Asensio Ramos, A. 2004, Nature, 430, 326

van Leeuwen, F. 2007, A\&A, 474, 653

Vögler, A., \& Schüssler, M. 2007, A\&A, 465, L43

Wedemeyer, S., Freytag, B., Steffen, M., Ludwig, H.-G., \& Holweger, H. 2004, A\&A, 414, 1121 